\title{
Differentiable Families of Stabilizers for Planar Bimodal Linear Control Systems
}

\author{
Josep Ferrer, M. Dolors Magret and Marta Peña \\ Departament de Matemàtica Aplicada I, ETSEIB, Universitat Politècnica de Catalunya, Diagonal 647, 08028 \\ Barcelona, Spain
}

\begin{abstract}
We consider bimodal linear control systems consisting of two subsystems acting on each side of a given hyperplane, assuming continuity along the separating hyperplane. For a differentiable family of controllable planar ones, we construct a differentiable family of feedbacks which pointwise stabilizes both subsystems.

Keywords: Controllability, parameterized families, feedback stabilization.

PACS: $02.10 . \mathrm{Ud}, 02.40 . \mathrm{Hw}, 07.05 . \mathrm{Dz}$
\end{abstract}

\section{INTRODUCTION}

Piecewise linear control systems (in particular, the bimodal ones: see, for example, [1], [2], [3]) have attracted the interest of the researchers in recent years by their wide range of applications, as well as by the possible theoretical approaches, even in the planar case (see, for example, [4]).

Bimodal linear control systems (BLCS) consist of two subsystems acting on each side of a given hyperplane, assuming continuity along the separating hyperplane. In the space of triples of matrices defining those systems, we consider the natural equivalence relation defined by changes of bases in the state space which preserves the hyperplanes parallel to the separating one.

Here we consider parameterized families of controllable planar BLCS, and we tackle the construction of parameterized families of stabilizers. In both cases, we say differentiable family when the involved matrices depend differentially on a parameter. Given a differentiable family of controllable BLCS, for each value of the parameter there is a feedback which stabilizes the corresponding system (see [3]). However, this parameterized family of pointwise stabilizers may not be differentiable (not even continuous). Our results allow to construct a differentiable family of feedbacks which stabilizes the corresponding system for each value of the parameter.

We point out that when dealing with parameterized families of BLCS, the non-generic case of unobservable ones appears in a natural way (see Example 1).

Throughout the paper, $\mathbb{R}$ will denote the set of real numbers, $M_{n \times m}(\mathbb{R})$ the set of matrices having $n$ rows and $m$ columns and entries in $\mathbb{R}$ (in the case where $n=m$, we will simply write $M_{n}(\mathbb{R})$ ).

\section{PRELIMINARIES}

A bimodal linear control system (BLCS) is given by two subsystems

$$
\left\{\begin{array}{l}
\dot{x}(t)=A_{1} x(t)+B_{1} u(t), \\
y(t)=C x(t),
\end{array} \quad \text { if } y(t) \leq 0, \quad\left\{\begin{array}{l}
\dot{x}(t)=A_{2} x(t)+B_{2} u(t), \\
y(t)=C x(t),
\end{array} \quad \text { if } y(t) \geq 0\right.\right.
$$

where $A_{1}, A_{2} \in M_{n}(\mathbb{R}) ; B_{1}, B_{2} \in M_{n \times 1}(\mathbb{R}) ; C \in M_{1 \times n}(\mathbb{R})$. One assumes that the dynamics is continuous along the separating hyperplane $H=\left\{x \in \mathbb{R}^{n}: C x=0\right\}$ and one can consider $C=\left(\begin{array}{lll}1 & 0 \ldots 0\end{array}\right) \in M_{1 \times n}(\mathbb{R})$. Hence $H=\left\{x \in \mathbb{R}^{n}\right.$ : $\left.x_{1}=0\right\}$ and continuity along $H$ is equivalent to:

$$
B_{2}=B_{1}, \quad A_{2} e_{i}=A_{1} e_{i}, \quad 2 \leq i \leq n .
$$

We will write from now on $B=B_{1}=B_{2}$.

We recall some definitions related to BLCS. 
Definition 1. The above BLCS is called observable if

$$
\operatorname{rank}\left(\begin{array}{c}
C \\
C A_{i} \\
\cdots \\
C A_{i}^{n-1}
\end{array}\right)=n, \quad i=1,2 .
$$

The above ranks are equal for both subsytems due to the continuity property of the system ([5]).

Definition 2. A BLCS is (completely) controllable if for any pair of states $\left(x_{0}, x_{f}\right)$ there exists a locally integrable input $u$ such that the solution $x^{x_{0}, u}$ passes through $x_{f}$, i.e. $x^{x_{0}, u}(T)=x_{f}$ for some $T>0$.

A well-known remarkable fact is that a single linear system $\dot{x}=A x+B u$ is controllable if and only if its 'controllability matrix' ( $\left.\begin{array}{llll}B & A B & \ldots & A^{n-1} B\end{array}\right)$ has maximal rank. For planar BLCS we recall the characterization of their controllability obtained in [1] for observable systems and generalized in [6] to unobservable ones:

Proposition 3. Let us consider a planar BLCS defined by $\left(A_{1}, A_{2}, B\right)$. We write $C_{1}, C_{2}$ the controllability matrices of both subsystems

$$
C_{1}=\left(\begin{array}{ll}
B & A_{1} B
\end{array}\right), \quad C_{2}=\left(\begin{array}{ll}
B & A_{2} B
\end{array}\right) .
$$

Then, it is controllable if and only if

$$
\operatorname{det} C_{1} \operatorname{det} C_{2}>0
$$

Remark 4. (1) Notice that, in particular, both subsystems must be controllable, but it is not a sufficient condition.

(2) Whereas for single systems the subset of controllable ones is open and dense, the above proposition shows that it is not for bimodal planar systems: controllability is an open, but not generic, property.

\section{FAMILIES OF STABILIZERS}

If the control function is a so-called 'feedback' of the type $u(t)=f(x(t))$, one obtains a dynamical system ('in closed loop'). In the linear case $\dot{x}=A x+B u$, a feedback $u=F x$ gives $\dot{x}=(A+B F) x$. A remarkable fact is that it is stable for some suitable $F$, provided that the initial control system be controllable.

As a natural generalization, in [3] any controllable planar BLCS is proved to be feedback stabilizable. Hence, if a differentiable parameterized family $\left(A_{1}(s), A_{2}(s), B(s)\right)$ is pointwise controllable (observable or not) then it is also pointwise stabilizable, that is to say, for any $s \in \mathbb{R}$ there is a common feedback $F(s)$ such that both closed-loop systems $A_{1}(s)+B(s) F(s), A_{2}(s)+B(s) F(s)$ are stable. However, the family $F(s)$ may not be differentiable (not even continuous). Here we prove that differentiable families of stabilizer feedbacks exist for $n=2$.

As we have pointed out in the Introduction, the unobservable case appears generically in parameterized families of BLCS. A typical case is considered in the following example. As an application of the above Proposition, we characterize when this family is pointwise controllable.

Example 1. Let us consider the parameterized family of planar BLCS

$$
A_{1}(s)=\left(\begin{array}{cc}
a_{1} & s \\
a_{2} & a_{4}
\end{array}\right), A_{2}(s)=\left(\begin{array}{cc}
\gamma_{1} & s \\
\gamma_{2} & a_{4}
\end{array}\right), B=\left(\begin{array}{l}
b_{1} \\
b_{2}
\end{array}\right)
$$

where $s \in \mathbb{R}$. Obviously, the systems defined by these matrices are observable except for $s=0$. Let us see that the family is pointwise controllable (i.e., for any $s \in \mathbb{R}$ the corresponding system is controllable) if and only if $b_{1} \neq 0$ and

(i) $a_{2} \gamma_{2}>0$, if $b_{2}=0$

(ii) $\operatorname{det}\left(\begin{array}{ll}a_{1} & b_{1} \\ a_{2} & b_{2}\end{array}\right)=\operatorname{det}\left(\begin{array}{ll}\gamma_{1} & b_{1} \\ \gamma_{2} & b_{2}\end{array}\right)$, otherwise.

From Proposition 3 and the analogous result in [1], for any $s \in \mathbb{R}$ (including the case where $s=0$ ) the corresponding system is controllable if and only if

$$
\left(b_{1}^{2} a_{2}+b_{1} b_{2} a_{4}-b_{1} b_{2} a_{1}-b_{2}^{2} s\right)\left(b_{1}^{2} \gamma_{2}+b_{1} b_{2} a_{4}-b_{1} b_{2} \gamma_{1}-b_{2}^{2} s\right)>0
$$


In particular $b_{1} \neq 0$ (it suffices to take $s=0$ ).

If $b_{2}=0$, the above inequality is

$$
\left(b_{1}^{2} a_{2}\right)\left(b_{1}^{2} \gamma_{2}\right)>0
$$

that is to say

$$
a_{2} \gamma_{2}>0
$$

Assume now $b_{2} \neq 0$. In general, two polynomials of degree 1 have the same sign at any point if and only if they have the same root and the slopes have the same sign. In our case both slopes are $-b_{2}^{2}$, so that the above inequality holds if and only if

$$
\frac{b_{1}^{2} a_{2}+b_{1} b_{2} a_{4}-b_{1} b_{2} a_{1}}{b_{2}^{2}}=\frac{b_{1}^{2} \gamma_{2}+b_{1} b_{2} a_{4}-b_{1} b_{2} \gamma_{1}}{b_{2}^{2}}
$$

which is equivalent (recall $b_{1} \neq 0$ ) to

$$
b_{1} a_{2}-b_{2} a_{1}=b_{1} \gamma_{2}-b_{2} \gamma_{1}
$$

Finally, we prove the existence of differentiable families of stabilizers for differentiable families of planar controllable bimodal systems.

\section{Proposition 5. Let}

$$
\left(A_{1}(s), A_{2}(s), B(s)\right), \quad s \in \mathbb{R}
$$

be a differentiable family of planar BLCS. If it is pointwise controllable, then there is a differentiable family of feedbacks $F(s)$, $s \in \mathbb{R}$, such that

$$
A_{1}(s)+B(s) F(s), \quad A_{2}(s)+B(s) F(s)
$$

are stable for any $s \in \mathbb{R}$.

Proof 1. Let us write

$$
\left.\begin{array}{c}
A_{1}(s)=\left(\begin{array}{ll}
a_{1} & a_{3} \\
a_{2} & a_{4}
\end{array}\right), A_{2}(s)=\left(\begin{array}{ll}
\gamma_{1} & a_{3} \\
\gamma_{2} & a_{4}
\end{array}\right), B(s)=\left(\begin{array}{l}
b_{1} \\
b_{2}
\end{array}\right) \\
C_{1}=\left(B(s) \quad A_{1}(s) B(s)\right), C_{2}=\left(B(s) \quad A_{2}(s) B(s)\right.
\end{array}\right)
$$

where all the coefficients are assumed differentiably depending on $s \in \mathbb{R}$. By hypothesis, we assume

$$
\operatorname{det} C_{1} \operatorname{det} C_{2}>0
$$

for any $s \in \mathbb{R}$. of

We look for $F(s)=\left(\begin{array}{lll}f_{1} & f_{2}\end{array}\right)$ where again we assume the coefficients depending on $s \in \mathbb{R}$, such that the eigenvalues

$$
\left(\begin{array}{ll}
a_{1}+b_{1} f_{1} & a_{3}+b_{1} f_{2} \\
a_{2}+b_{2} f_{1} & a_{4}+b_{2} f_{2}
\end{array}\right),\left(\begin{array}{ll}
\gamma_{1}+b_{1} f_{1} & a_{3}+b_{1} f_{2} \\
\gamma_{2}+b_{2} f_{1} & a_{4}+b_{2} f_{2}
\end{array}\right)
$$

have negative real part for any $s \in \mathbb{R}$ or, equivalently, the matrices have negative trace and positive determinant, that is to say:

$$
\begin{gathered}
b_{1} f_{1}+b_{2} f_{2}<-a_{1}-a_{4}, \\
b_{1} f_{1}+b_{2} f_{2}<-\gamma_{1}-a_{4}, \\
f_{1}\left(a_{3} b_{2}-a_{4} b_{1}\right)+f_{2}\left(a_{2} b_{1}-a_{1} b_{2}\right)<a_{1} a_{4}-a_{2} a_{3}, \\
f_{1}\left(a_{3} b_{2}-a_{4} b_{1}\right)+f_{2}\left(\gamma_{2} b_{1}-\gamma_{1} b_{2}\right)<\gamma_{1} a_{4}-\gamma_{2} a_{3} .
\end{gathered}
$$

We change the variables $\left(f_{1}, f_{2}\right)$ by $(x, y)$ defined by

$$
\begin{aligned}
& x=b_{1} f_{1}+b_{2} f_{2}, \\
& y=\left(b_{2} a_{3}-b_{1} a_{4}\right) f_{1}+\left(b_{1} a_{2}-b_{2} a_{1}\right) f_{2},
\end{aligned}
$$

which is a change of variables, because (by hypothesis):

$$
\operatorname{det}\left(\begin{array}{cc}
b_{1} & b_{2} a_{3}-b_{1} a_{4} \\
b_{2} & b_{1} a_{2}-b_{2} a_{1}
\end{array}\right)=\operatorname{det} C_{1} \neq 0 .
$$


Then:

$$
f_{1}=\frac{\left(b_{1} a_{2}-b_{2} a_{1}\right) x-b_{2} y}{\operatorname{det} C_{1}}, \quad f_{2}=-\frac{\left(b_{2} a_{3}-b_{1} a_{4}\right) x-b_{1} y}{\operatorname{det} C_{1}} .
$$

With this change of variables, the desired inequalities become:

$$
\begin{gathered}
x<-a_{1}-a_{4}, \\
x<-\gamma_{1}-a_{4}, \\
y<a_{1} a_{4}-a_{2} a_{3}, \\
\left(a_{3} b_{2}-a_{4} b_{1}\right) \frac{\left(b_{1} a_{2}-b_{2} a_{1}\right) x-b_{2} y}{\operatorname{det} C_{1}}-\left(\gamma_{2} b_{1}-\gamma_{1} b_{2}\right) \frac{\left(b_{2} a_{3}-b_{1} a_{4}\right) x-b_{1} y}{\operatorname{det} C_{1}}<\gamma_{1} a_{4}-\gamma_{2} a_{3} .
\end{gathered}
$$

In order to see that there exist solutions $(x, y)$, it is sufficient that some of the coefficients of the variables $x$, $y$ are positive. For the last one we have:

$$
-\frac{b_{2}\left(a_{3} b_{2}-a_{4} b_{1}\right)}{\operatorname{det} C_{1}}+\frac{b_{1}\left(\gamma_{2} b_{1}-\gamma_{1} b_{2}\right)}{\operatorname{det} C_{1}}=\frac{-a_{3} b_{2}^{2}+a_{4} b_{1} b_{2}+\gamma_{2} b_{1}^{2}-\gamma_{1} b_{1} b_{2}}{\operatorname{det} C_{1}}=\frac{\operatorname{det} C_{2}}{\operatorname{det} C_{1}}>0
$$

again by the hypothesis.

\section{REFERENCES}

1. Camlibel, K., Heemels, M. \& Schumacher, H., Stability and controllability of planar bimodal linear complementarity systems, Proceedings of the 42nd IEEE Conference on Decision and Control, 1651-1656, 2003.

2. Camlibel, K., Heemels, M. \& Schumacher, H., On the controllability of bimodal piecewise linear systems, LNCS 2993, 250-264, 2004.

3. Camlibel, K., Heemels, M. \& Schumacher, H., A full characterization of stabilizability of bimodal piecewise linear systems with scalar inputs, Automatica, 44, 1261-1267, 2008.

4. Di Bernardo, M., Pagano, D. J. \& Ponce, E., Nonhyperbolic boundary equilibrium bifurcations in planar Filippov systems: a case study approach, J. Bifur. Chaos Appl. Sci. Engin., 18, 1377-1392, 2008.

5. Ferrer, J., Magret, M. \& PeÑa, M., Bimodal piecewise linear systems. Reduced forms, International Journal of Bifurcation and Chaos, 20, 2795-2808, 2010.

6. Ferrer, J., PaCha, J. R. \& PeÑA, M., Controllability of continuous bimodal linear systems, Mathematical Problems in Engineering (special issue Mathematical Modeling, Analysis, and Control of Hybrid Dynamical Systems), Volume 2013 (2013), Article ID 342548, 14 pages. 